\title{
Violencia física contra la mujer en Santa Fe de Bogotá: prevalencia y factores asociados ${ }^{1}$
}

\author{
Joanne Klevens ${ }^{2}$
}

RESUMEN Objetivos. Estimar la magnitud del problema de la violencia conyugal que afecta a las mujeres que acuden a los servicios de salud pública en Santa Fe de Bogotá, Colombia, e identificar los factores asociados con el riesgo de ser agredidas físicamente.

Métodos. Se analizaron los datos recolectados desde septiembre de 1998 hasta septiembre de 1999 mediante entrevistas de las 3971 mujeres con niños menores de 6 meses de edad, que solicitaron consulta por diversas causas pediátricas (control del niño sano, vacunación o morbilidad del menor) o fueron atendidas por parto en la red de servicios de salud pública en la localidad de Suba, Santa Fe de Bogotá, Colombia. De la muestra indicada, 10 mujeres rehusaron participar y 804 no tenían compañero, por lo cual se excluyeron de los análisis.

Resultados. De las 3157 participantes que convivían con un compañero íntimo, 26,5\% informaron que habían sido abofeteadas o empujadas por su compañero actual y 13,3\% que habían sido agredidas gravemente por medio de golpes con el puño cerrado, patadas, golpes con algún objeto, "golpizas" y amenazas con un cuchillo o arma de fuego. Además de lo anterior, $26,2 \%$ de ellas indicaron que su compañero les imponía alguna prohibición. En los análisis bivariados, la violencia se asoció significativamente con menos escolaridad y menores ingresos $(\mathrm{P}<0,001)$, mayor número de hijos $(\mathrm{P}<0,001)$, mayor tiempo de unión con el compañero actual $(\mathrm{P}<0,001)$, mayor frecuencia de conflicto con la pareja $(\mathrm{P}<0,001)$, ausencia de otros familiares en el hogar $(\mathrm{P}<0,01)$, historia de maltrato en la familia de origen $(\mathrm{P}<0,001)$ y prohibiciones por parte del compañero $(\mathrm{P}<0,001)$. La frecuencia del conflicto con la pareja y las prohibiciones fueron los factores más fuertemente asociados con la violencia, según el análisis multivariado.

Conclusiones. Los servicios de salud pública son un buen lugar para detectar a las víctimas de la violencia intrafamiliar. Deben establecerse en ellos programas de detección precoz y de intervenciones efectivas. Los servicios de apoyo y protección de las víctimas tienen que extenderse y fortalecerse para prestarles orientación, asistencia jurídica, y capacitación laboral y educativa. También es importante desarrollar servicios alternativos apropiados para los agresores.

Palabras clave Violencia intrafamiliar, maltrato de la mujer, salud maternoinfantil.

La violencia contra la mujer, especialmente por parte de sus compañe-

\footnotetext{
1 Trabajo financiado por la Secretaría Distrital de Salud, Santa Fe de Bogotá, Colombia.

2 Consultora e investigadora independiente en epidemiología y prevención de la violencia. Dirección para correspondencia: Calle 106B \#40A-12, Santa Fe de Bogotá, Colombia. Correo electrónico: jklevens@ colnodo.apc.org
}

ros íntimos, se ha señalado como un problema prioritario de salud pública por su magnitud y gravedad, y por la carga que representa para los servicios de salud en función de los costos de atención que genera (1). Esta violencia no solo causa daños físicos y psicológicos a las mujeres sino que también implica riesgos para sus hijos. Presenciar la violencia conyugal incrementa en los niños las probabilidades de sufrir depresión, ansiedad, trastornos de conducta y retrasos en su desarrollo cognoscitivo (2). Además, aumenta el riesgo de convertirse a su vez en víctimas de maltrato (3).

En la región de América Latina y el Caribe, cada vez hay mayor conciencia 
del problema y se han hecho esfuerzos loables para ofrecer servicios a las víctimas e introducir sanciones judiciales contra sus agresores (4). Sin embargo, las acciones para su prevención y control son todavía muy limitadas (5).

En las revisiones sistemáticas de la literatura se identifican algunos factores asociados comúnmente con la violencia conyugal, por ejemplo, la frecuencia de conflicto entre la pareja, el bajo estrato social, haber sido testigos de la violencia en su familia de origen y el consumo excesivo de alcohol (6-8). Lamentablemente, estos hallazgos se basan en su gran mayoría en poblaciones desarrolladas de habla inglesa, de manera que es poco lo que se sabe sobre el problema en otros medios socioculturales.

Los pocos estudios sobre este tema que se han realizado en América Latina corroboran la importancia que tienen el bajo estrato social, la historia de maltrato en la familia de origen y el consumo excesivo de alcohol como factores de riesgo para la violencia conyugal (9-10). En Nicaragua, Ellsberg et al. encontraron además que las prohibiciones de trabajar, estudiar, recibir visitas, visitar a la familia o las amistades, o planificar la familia impuestas por el compañero a la mujer incrementaban fuertemente el riesgo de violencia física contra ella (10).

En este trabajo se intentó estimar la magnitud del problema de la violencia conyugal que afectaba a una muestra de las mujeres que acudieron a consulta durante un año en los servicios de salud pública de Santa Fe de Bogotá, Colombia, e identificar los factores que aumentaban el riesgo de ser objeto de agresión física. En último término, se deseaba contribuir con elementos apropiados al desarrollo de programas de control.

\section{MATERIALES Y MÉTODOS}

Los datos utilizados en este trabajo se obtuvieron en entrevistas realizadas para identificar a las madres en alto riesgo de maltratar a sus hijos, con el fin de ofrecer una intervención pre- ventiva y evaluar la efectividad de esta. Durante el proyecto se abordaron todas las madres tratadas en los servicios de posparto o que tenían niños menores de 6 meses de edad y acudieron a consulta pediátrica para control del niño sano, vacunación o tratamiento del lactante a la red de servicios de salud pública en la localidad de Suba, Santa Fe de Bogotá, Colombia, entre septiembre de 1998 y septiembre de 1999. En total se contactaron 3971 mujeres a las cuales se les informó de los objetivos del estudio. Se les aseguró asimismo que la información que ofrecieran sería confidencial y que tenían el derecho de rehusar contestar cualquier pregunta de la entrevista. Diez mujeres no quisieron o pudieron participar por limitaciones del idioma o del tiempo, o traslado a otro servicio obstétrico.

Todas las participantes fueron entrevistadas en un lugar privado por una mujer previamente entrenada. Se usó un cuestionario estandarizado. En la entrevista se obtuvo información sobre la edad y escolaridad de la mujer, el ingreso familiar, el número de hijos, la historia de maltrato en su familia de origen, la convivencia con familiares y compañeros íntimos, la frecuencia de los conflictos con el compañero actual y sus experiencias de victimización. Además, sobre la base del estudio nicaragüense (10), se exploraron las prohibiciones impuestas por sus compañeros íntimos en relación con las actividades sociales, el trabajo o la planificación familiar. Si bien dichas prohibiciones son otra forma de agresión, en este estudio se analizaron solo como factor de riesgo para la agresión física.

Se utilizaron los ocho elementos de agresión física de la escala de resolución de conflictos (Conflict Tactics Scale) de Strauss (11) para establecer la prevalencia y la gravedad de la violencia generada por el compañero actual durante todo el período de convivencia. Además de los ítemes de agresión física, la escala de resolución de conflictos contiene una escala de agresión verbal y otra de negociación, las cuales no se usaron en este estudio. La escala de Strauss es el instrumento más ampliamente utilizado en estudios sobre violencia doméstica, pero ha sido criticada por limitarse a los actos específicos de violencia sin considerar el contexto, la intencionalidad, la historia previa de violencia, los factores precipitantes ni la secuencia de eventos; por sus definiciones arbitrarias de agresión moderada y severa; y por múltiples problemas de confiabilidad y validez (12).

De las mujeres entrevistadas, 804 $(20,3 \%)$ indicaron que en el momento no convivían con un compañero íntimo. Como se explicó anteriormente, los datos para este trabajo se tomaron de información adquirida en un proyecto de prevención de maltrato a los niños. Dado que el interés de la entrevista en ese proyecto era identificar a las mujeres en alto riesgo de maltratar a sus hijos, para ello interesaba conocer la posible situación de violencia infligida por su compañero actual como factor de riesgo. No se incluyeron preguntas sobre su historia de victimización por compañeros anteriores. Por esta razón, las 804 mujeres sin compañero actual no aportaron ese tipo de información y fueron excluidas de los análisis del presente trabajo.

Toda la información recolectada se sistematizó y procesó mediante el paquete estadístico Statistical Package for the Social Sciences. Se estableció la prevalencia de cada tipo de agresión y luego, sobre la base de las categorías establecidas por Strauss (11), se clasificaron actos de agresión moderada empujar, abofetear y arrojar objetos; y de agresión grave, golpes con el puño cerrado, patadas, golpes con algún objeto, "golpizas" y amenazas con cuchillo o arma de fuego. Las diferencias de prevalencia entre estos dos tipos de maltrato se compararon para cada factor explorado mediante la razón de riesgos $(R R)$ y la prueba de ji al cuadrado $\left(\chi^{2}\right)$.

Por último, las variables que resultaron significativas en los análisis bivariados se convirtieron en variables dicotómicas (excepto la escolaridad, que se clasificó en tres categorías) para introducirlas en un modelo de regresión 
logística con el fin de establecer, tanto para la agresión moderada como para la agresión grave, el grado de asociación de cada factor después de controlar la influencia de las demás variables. Se analizaron también modelos de regresión logística estratificando por prohibiciones (frente a no prohibiciones) y luego por nivel de conflicto con el fin de establecer las posibles interacciones entre estas dos variables.

\section{RESULTADOS}

Las 3157 mujeres entrevistadas que declararon convivir con un compañero íntimo tenían entre 14 y 46 años de edad, con una media de 24,9 años. Además tenían, en promedio, 7,6 años de estudio formal. De ellas, $74,7 \%$ tenían uno o dos hijos y $91 \%$ vivían en un hogar con otros familiares además de los hijos y la pareja. El ingreso familiar total de $63 \%$ era igual a un salario mínimo (US\$ 145) o menor. Más de la mitad de ellas $(52,6 \%)$ llevaban menos de un año conviviendo con su pareja actual.

En este grupo de mujeres, $27,3 \%$ informaron que habían sido víctimas de algún tipo de agresión por parte de su compañero actual. De ellas, $67,4 \%$ dijeron que habían sido objeto de dos ó mas tipos de agresión física; $26,5 \%$ habían sido abofeteadas o empujadas (agresión moderada) por su compañero actual $-58 \%$ de las dos maneras- y $13,3 \%$ habían sido agredidas gravemente, $-46,7 \%$ de dos o más formas (cuadro 1) - Tanto la agresión moderada como la grave se asoció significativamente con menos escolaridad e ingresos $(P<0,001)$, un mayor número de hijos $(P<0,001)$ y mayor tiempo de unión con el compañero actual $(P<0,001)$ (cuadro 2). A su vez, a mayor frecuencia de conflicto con la pareja, mayor el riesgo de victimización $(P<0,001)$. Las mujeres que no tenían a otros familiares en el hogar estaban también en mayor riesgo de ser agredidas físicamente $(P<0,01)$ por su compañero.

Además de lo anterior, $26,2 \%$ de las mujeres revelaron que su compañero les prohibía ciertas actividades: a 14\%

CUADRO 1. Porcentaje de una muestra de 3157 mujeres que declararon haber sufrido distintos tipos de agresión física por parte de su compañero actual. Suba, Bogotá, septiembre de 1998 a septiembre de 1999

\begin{tabular}{lc}
\hline \multicolumn{1}{c}{$\begin{array}{c}\text { Tipo de agresión cometida } \\
\text { por el compañero }\end{array}$} & Porcentaje \\
\hline Le tiró algo alguna vez & 7,5 \\
La empujó o sacudió alguna vez & 22,7 \\
La abofeteó alguna vez & 18,4 \\
Le tiró algo, la empujó o la abofeteó & 26,5 \\
(violencia moderada) & 11,9 \\
La pateó o le pegó con el puño & 3,3 \\
La golpeó con un objeto & 5,4 \\
Le dio una golpiza & 2,1 \\
La amenazó con APa o AFb & 0,6 \\
La hirió con AP o AF & \\
La pateó, la golpeó con objeto, le dio una golpiza, & 13,3 \\
la amenazó o la hirió con un arma & \\
(violencia grave) & \\
\hline a AP = arma punzante. & \\
${ }^{b} \mathrm{AF}=$ arma de fuego. &
\end{tabular}

les estaba prohibido trabajar, estudiar o visitar a sus amigas; a 10\%, recibir visitas en la casa; a $8 \%$, visitar a su familia; y a $3 \%$, planificar sus embarazos. Todas estas prohibiciones duplicaban o triplicaban el riesgo de agresión física. A $10 \%$ de las mujeres se les habían impuesto dos o más prohibiciones, lo cual incrementó alrededor de tres veces el riesgo de ser empujadas o abofeteadas por su compañero y cinco veces el de ser agredidas gravemente.

En cuanto a experiencias en su familia de origen, $64,4 \%$ de las mujeres indicaron haber recibido algún tipo de maltrato durante su niñez: en $34,6 \%$, el maltrato fue emocional; en $43 \%$, maltrato físico leve; en 57,9\%, maltrato físico moderado; y en $12,2 \%$, maltrato físico grave. Todos los tipos de maltrato recibidos en la niñez incrementaron el riesgo de ser agredidas en la relación conyugal. No obstante, al controlar la influencia de las demás variables, el maltrato durante la niñez no se asoció significativamente con el riesgo de agresión física grave $(P<0,43)$ pero sí se asoció débilmente con agresión física leve $(P<0,001)$ (cuadro 3 ).

Los resultados del análisis multivariado mostraron también que la frecuencia del conflicto con la pareja y las prohibiciones de parte del compañero son los factores más fuertemente aso- ciados con el riesgo de ser víctima de agresión física, independientemente de los ingresos, la escolaridad, el número de hijos y el tiempo de convivencia como pareja (cuadro 3). No parece haber una interacción entre las prohibiciones y el conflicto, pues la magnitud de las asociaciones con agresión moderada y grave fueron similares al analizar los datos estratificados por estas variables.

\section{DISCUSIÓN}

Los datos obtenidos en la muestra de mujeres descrita indican que casi la tercera parte de ellas convivían en ese momento con un compañero que las agredía físicamente. Debe tenerse en cuenta que las mujeres que acuden a los servicios de salud públicos tienden a pertenecer a un estrato socioeconómico bajo, lo cual a su vez se asocia con la violencia conyugal (9-10). Por lo tanto, es posible que las cifras obtenidas sean más altas de lo que se observaría en la población en general. Por otro lado, deben interpretarse con cautela, ya que se basan en autoinformes sin la confirmación de otras fuentes. Es posible que a las entrevistadas se les hubiera olvidado la incidencia de algún tipo de agresión o que prefirie- 
CUADRO 2. Riesgo relativo (RR) e intervalo de confianza de $95 \%$ (IC95\%) de sufrir violencia física moderada o grave a manos del compañero actual, según las características demográficas y familiares de 3157 mujeres entrevistadas. Suba, Bogotá, septiembre de 1998 a septiembre de 1999

\begin{tabular}{|c|c|c|}
\hline \multirow{2}{*}{$\begin{array}{l}\text { Característica demográfica } \\
\text { o familiar de las mujeres }\end{array}$} & $\begin{array}{l}\text { Agresión física } \\
\text { moderada }\end{array}$ & $\begin{array}{l}\text { Agresión física } \\
\text { grave }\end{array}$ \\
\hline & $R R(\mathrm{IC} 95 \%)$ & $R R(\mathrm{IC} 95 \%)$ \\
\hline \multicolumn{3}{|l|}{ Edad (en años) } \\
\hline$<18$ & $1,14(0,94-1,38)$ & $1,11(0,77-1,59)$ \\
\hline $18-34$ & 1,00 & 1,00 \\
\hline$\geq 35$ & $1,12(0,91-1,37)$ & $1,52(1,16-1,99)$ \\
\hline \multicolumn{3}{|l|}{ Escolaridad (en grados) } \\
\hline$<6^{\circ}$ & $2,35(19,2-2,87)$ & $3,32(2,41-4,58)$ \\
\hline $6^{\circ}-10^{\circ}$ & $1,73(1,46-2,06)$ & $2,17(1,64-2,88)$ \\
\hline$\geq 11^{\circ}$ & 1,00 & 1,00 \\
\hline \multicolumn{3}{|l|}{ Ingreso (en salarios mínimos) } \\
\hline $0-1$ & $3,23(2,48-4,21)$ & $4,51(2,90-6,99)$ \\
\hline 2 & $1,67(1,23-2,26)$ & $1,58(0,94-2,65)$ \\
\hline$\geq 3$ & 1,00 & 1,00 \\
\hline Vivir sin familiares & $2,06(1,81-2,36)$ & $2,47(2,01-3,05)$ \\
\hline \multicolumn{3}{|l|}{ No. de hijos } \\
\hline 1 & 1,00 & 1,00 \\
\hline $2-3$ & $1,58(1,37-1,81)$ & $1,94(1,54-2,44)$ \\
\hline$\geq 4$ & $2,09(1,75-2,50)$ & $3,70(2,84-4,80)$ \\
\hline \multicolumn{3}{|l|}{ Tiempo de convivencia } \\
\hline$<1$ año & 1,00 & 1,00 \\
\hline$>1$ año & $1,91(1,68-2,17)$ & $2,26(1,85-2,77)$ \\
\hline \multicolumn{3}{|l|}{ Frecuencia de conflicto conyugal } \\
\hline$<5$ veces al año & 1,00 & 1,00 \\
\hline 5-11 veces al año & $9,10(5,94-13,93)$ & $14,07(6,04-32,72)$ \\
\hline $1-5$ veces al mes & $13,37(8,87-20,15)$ & $21,83(9,61-49,62)$ \\
\hline $1-2$ veces a la semana & $19,35(12,88-29,08)$ & $39,23(17,38-88,57)$ \\
\hline Casi todos los días & $27,65(18,49-41,34)$ & $74,03(33,06-165,78)$ \\
\hline \multicolumn{3}{|l|}{ Prohibiciones impuestas por el compañero } \\
\hline Trabajar o estudiar & $2,09(1,86-2,36)$ & $2,78(2,32-3,33)$ \\
\hline Visitar amigas & $2,48(2,22-2,77)$ & $3,66(3,09-4,34)$ \\
\hline Visitar familia & $2,95(2,65-3,29)$ & $4.63(3,90-5,48)$ \\
\hline Recibir visitas & $2,64(2,35-2,95)$ & $3,27(2,73-3,93)$ \\
\hline Usar métodos de planificación familiar & $2,13(1,76-2,57)$ & $2,95(2,24-3,88)$ \\
\hline Cualquier prohibición & $2,75(2,48-3,09)$ & $3,70(3,10-4,41)$ \\
\hline A más de dos actividades & $3,32(2,95-3,73)$ & $5,10(4,24-6,13)$ \\
\hline \multicolumn{3}{|l|}{ Historia de maltrato en la familia de origen } \\
\hline Insultos, rechazo, descuido & $2,26(2,02-2,54)$ & $2,68(2,24-3,20)$ \\
\hline Tirar objetos, empujar, pegar con la mano & $2,14(1,90-2,41)$ & $2,13(1,78-2,56)$ \\
\hline Golpes con objeto o puño & $1,78(1,56-2,04)$ & $1,66(1,37-2,03)$ \\
\hline Golpizas, quemaduras, requirió atención médica & $1,85(1,62-2,11)$ & $2,48(2,05-3,01)$ \\
\hline Cualquier tipo de maltrato & $1,97(1,70-2,29)$ & $1,90(1,53-2,37)$ \\
\hline
\end{tabular}

ran no declararlo por miedo o vergüenza. Además, se exploró solo la agresión física por parte del compañero actual y el instrumento utilizado (la escala de resolución de conflictos) adolece de problemas de confiabilidad y validez. No obstante, las cifras encontradas en esta población aportan un testimonio adicional de la frecuencia de la violencia conyugal a la que es- taban sometidas las mujeres que acudieron a los servicios de salud pública durante nuestro estudio.

Los resultados aquí presentados son comparables con los de otros estudios. Las encuestas nacionales indican que, en Colombia, alrededor de $20 \%$ de las mujeres declaran haber sido golpeadas alguna vez por su cónyuge (13). En cuanto a América Latina en general, los estudios disponibles registran cifras de violencia conyugal que afectan a entre 20 y $60 \%$ de las mujeres encuestadas (1).

Nuestros datos corroboran la importancia del conflicto conyugal, la historia de maltrato en la familia de origen, el bajo estrato socioeconómico y el menor grado de escolaridad como marcadores de riesgo (8-9). Además se 
encontró que entre los factores más fuertemente asociados con el riesgo de victimización figuraban las prohibiciones por parte del compañero. Esto tiende a mostrar una vez más que una parte importante de la violencia conyugal contra la mujer ocurre dentro de un contexto de control y dominación (12). El diseño transversal de este estudio no permite establecer la dirección ni la relación causal con estos factores. No obstante, los resultados permiten sugerir algunas acciones para la prevención y el control de la violencia física contra la mujer.

Primero, podría ser importante alertar a las mujeres sobre los posibles riesgos de permanecer en una relación conflictiva o bajo el control excesivo de su compañero, para que puedan tomar mejores decisiones frente a sus relaciones de pareja. Sin embargo, es necesario que futuras investigaciones aclaren la secuencia o historia natural de las prohibiciones y del conflicto en la violencia conyugal para saber si en realidad son precursores de la agresión fisica. De resultar así, estos dos factores también podrían ayudar a identificar precozmente a las mujeres en mayor riesgo con el fin de ofrecerles una intervención oportuna.

En segundo lugar, la frecuencia de la violencia contra las mujeres que acudieron a los servicios de salud pública durante el año de estudio y las múltiples consecuencias para su salud física y mental ubican a estos servicios en una posición estratégica para identificar e intervenir en el problema. Esto es de especial importancia cuando las mujeres tienen hijos — como las de

CUADRO 3. Riesgo relativo indirecto (RRI) e intervalo de confianza de $95 \%$ (IC95\%) observados en los modelos de regresión logística para la violencia física moderada o grave infligida por el compañero actual, según las características demográficas y familiares de 3157 mujeres. Suba, Bogotá, septiembre de 1998 a septiembre de 1999

\begin{tabular}{|c|c|c|}
\hline \multirow{2}{*}{$\begin{array}{l}\text { Característica demográfica } \\
\text { o familiar de las mujeres }\end{array}$} & $\begin{array}{c}\text { Agresión física } \\
\text { moderada }\end{array}$ & $\begin{array}{c}\text { Agresión física } \\
\text { grave }\end{array}$ \\
\hline & $R R I(\mathrm{IC} 95 \%)$ & $R R I(\mathrm{IC} 95 \%)$ \\
\hline Escolaridad $<6$ grados & $1,52(1,33-1,71)$ & $1,54(1,30-1,77)$ \\
\hline $6-10$ grados & $1,23(1,04-1,45)$ & $1,24(1,01-1,53)$ \\
\hline Ingresos $0-1$ salarios mínimos & $1,70(1,36-2,11)$ & $2,14(1,56-2,92)$ \\
\hline$\geq 3$ hijos & $1,24(1,12-1,37)$ & $1,45(1,29-1,63)$ \\
\hline Tiempo de convivencia >1 año & $1,79(1,42-2,59)$ & $1,63(1,18-2,27)$ \\
\hline Conflicto conyugal 1 ó más veces/semana & $4,26(3,53-5,15)$ & $4,60(3,63-5.84)$ \\
\hline Prohibiciones impuestas por el compañero & $2,97(2,45-3,60)$ & $2,96(2,33-3,75)$ \\
\hline Historia de maltrato en la familia de origen & $1,25(1,12-1,39)$ & $1,07(0,93-1,23)$ \\
\hline \% que clasifica correctamente el modelo & $77,8 \%$ & $87,6 \%$ \\
\hline$X^{2}$, grados de libertad y $P$ del modelo & 724,$7 ; 7 ; 0,0000$ & 532,$2 ; 7 ; 0,0000$ \\
\hline
\end{tabular}

nuestra población de estudio-, por los riesgos a que ellos también están expuestos. Hay varios instrumentos más sencillos que la escala de resolución de conflictos utilizada en este estudio, que tienen buena sensibilidad y especificidad para identificar a las mujeres víctimas de violencia en los servicios de salud (15-16). Aunque algunos trabajadores de la salud son reacios a indagar sobre la posibilidad de maltrato, en este trabajo, al igual que en otros, $(10,14)$ se encontró que las mujeres entrevistadas estaban muy dispuestas a cooperar y agradecidas por el interés en su bienestar.

Varias asociaciones médicas han formulado recomendaciones para el manejo de la mujer que es víctima de la violencia conyugal (17-20). Por el carácter repetitivo de esta violencia y las pocas probabilidades de que desapa- rezca espontáneamente, su detección precoz y manejo efectivo no solo podrían evitarle una multiplicidad de problemas a la mujer, sino que además serían estrategias para prevenir el maltrato de los niños.

Para establecer un programa de detección precoz, será necesario ofrecer entrenamiento especial a los trabajadores de la salud para que aprendan a reconocer el problema, lo manejen con sensibilidad y conozcan las opciones de acción. Por supuesto, es primordial disponer de programas de intervención efectiva. Los servicios de apoyo y protección de las víctimas tienen que extenderse y fortalecerse para prestarles orientación, asistencia legal, y capacitación laboral y educativa. Por otra parte, deben desarrollarse servicios alternativos efectivos para los agresores.

\section{REFERENCIAS}

1. Heise L, Raikes A, Watts $\mathrm{CH}$, Zwi AB. Violence against women: a neglected public health issue in less developed countries. Soc Sci Med 1994; 39(9):1165-1179.

2. Fantuzzo JW, Lindquist CU. The effects of observing conjugal violence on children: a review and analysis of research methodology. J Fam Viol 1989;4(1):77-94.

3. Orpinas P. Who is violent?: factors associated with aggressive behaviors in Latin America and Spain. Rev Panam Salud Publica 1999; 5(4-5):232-244.

4. Shrader E, Sagot, M. Domestic violence: women's way out. Washington, DC: Pan American Health Organization; 2000. (Occasional Publication No. 2).

5. Larrain, S. Violencia doméstica contra la mujer en América Latina y el Caribe: revisión de dos décadas de acción. Trabajo presentado a la conferencia Violencia Doméstica en Amé- rica Latina y el Caribe patrocinada por el Banco Interamericano de Desarrollo. Washington, DC: 20 a 21 de octubre de 1997.

6. Hotaling GT, Sugarman DB. An analysis of risk markers in husband to wife violence: the current state of knowledge. Viol Vict 1986; 1(2):101-124.

7. Tolman RM, Bennett LW. A review of quantitative research on men who batter. J Interpers Viol 1990;5(1);87-118. 
8. Hotaling GT, Sugarman DB. A risk marker analysis of assaulted wives. J Fam Viol 1990; 5(1):1-13.

9. Moreno Martín F. La violencia en la pareja. Rev Panam Salud Pública 1999;5(4-5): 245-258.

10. Ellsberg M, Peña R, Herrera A, Liljestrand J, Winkvist A. Confites en el infierno: prevalencia y características de la violencia conyugal hacia las mujeres en Nicaragua. Managua: Asociación de Mujeres Profesionales; 1996.

11. Strauss MA. Measuring intrafamily conflict and violence: The Conflict Tactics (CT) Scale. J Marr Fam 1979;41(2):75-88.

12. Dobash RP, Dobash RE, Wilson M, Daly M. The myth of sexual symmetry in marital violence. Soc Prob 1992;39(1):71-91.
13. Profamilia. Encuesta nacional de demografía y salud, 1995. Santa Fe de Bogotá: Profamilia; 1995.

14. Friedman LS, Samet JH, Roberts MS, Hudlin $M$, Hans P. Inquiry about victimization experiences: a survey of patient preferences and physician practices. Arch Intern Med 1992; 152 (6):1186-1190.

15. Norton LB, Peipert JF, Zierler S, Lima B, Hume L. Battering in pregnancy: an assessment of two screening methods. Obstet Gynecol 1995;85(3):321-325.

16. Sherin KM, Sinacore JM, Li XQ, Zitter RE, Shakil A. HITS. A short domestic violence screening tool for use in a family practice setting. Fam Med 1998;30(7):508-512.

17. American Medical Association, Council on Scientific Affairs. Violence against women: relevance for medical practitioners. JAMA 1992;267(23):3184-3189.

18. American College of Obstetricians and Gynecologists (ACOG). The battered woman. Washington, DC: ACOG; 1995. (Technical Bulletin 209).

19. American Academy of Pediatrics. The role of the pediatrician in recognizing and intervening on behalf of abused women. Pediatrics 1998;101(6):1091-1092.

20. Bishop J, Patterson PGR. Guidelines for the evaluation and management of family violence. Can J Psychiatry 1992;37(7):458-471.

Manuscrito recibido el 13 de julio de 2000. Aceptado, tras revisión, el 27 de diciembre de 2000.

ABSTRACT Objective. Estimate the magnitude of the problem of violence in intimate relationships affecting women in Santa Fe de Bogotá, Colombia, and identify the factors related to the risk of being battered.

Physical violence against Methods. The data analyzed were collected between September 1998 and September 1999 from interviews with 3971 women who had a child less than 6 months old. The women interviewed were ones using public health services in the Suba area of Bogotá, either for pediatric attention (well-baby services, vaccination, an ill child) or for childbirth. Out of the 3971 women, 10 of them declined to participate, and 804 of them were not in an intimate relationship and were excluded from the analyses.

Results. Of the 3157 participants, $26.5 \%$ of them reported that their current partner had slapped or pushed them, and $13.3 \%$ reported they had been hit with a fist, kicked, hit with some object, or beaten, or threatened with a knife or gun. In addition, $26.2 \%$ of the women said that their partner imposed some prohibition on them (on social activities, work, family planning, etc.). In the bivariate analyses, violence was significantly associated with: less schooling and lower income $(P<0.001)$, having more children $(P<0.001)$, a longer period of time living with the partner $(P<0.001)$, more frequent conflict with the partner $(P<0.001)$, not having other family members living in the home $(P<0.01)$, a history of abuse in the family of origin $(P<0.001)$, and prohibitions imposed by the partner $(P<0.001)$. According to multivariate analysis, the two factors most strongly related with violence were frequency of conflict with the partner and prohibitions imposed by the partner.

Conclusions. Public health services are a good place to identify victims of domestic violence, and early detection and intervention programs should be established there. Services to support and protect victims should be expanded and strengthened in order to provide those persons with guidance, legal assistance, education, and job training. Appropriate alternative services for aggressors also need to be developed. 